\title{
Association of Monocyte Chemoattractant Protein-1 with Death and Atherosclerotic Events in Chronic Kidney Disease
}

\author{
L. Parker Gregg ${ }^{a, b} \quad$ Maria Clarissa Tio ${ }^{c} \quad$ Xilong Lid $^{d} \quad$ Beverley Adams-Huet ${ }^{d}$ \\ James A. de Lemos ${ }^{\mathrm{e}}$ S. Susan Hedayati ${ }^{\mathrm{a}}$ \\ ${ }^{a}$ Division of Nephrology, Department of Medicine, University of Texas Southwestern Medical Center, Dallas, \\ TX, USA; ${ }^{b}$ Division of Nephrology, Medical Service, Veterans Affairs North Texas Health Care System, Dallas, TX, \\ USA; ' Department of Medicine, University of Texas Southwestern Medical Center, Dallas, TX, USA; ${ }^{\mathrm{d}}$ Division of \\ Biostatistics, Department of Clinical Sciences, University of Texas Southwestern Medical Center, Dallas, TX, USA; \\ e Division of Cardiology, Department of Medicine, University of Texas Southwestern Medical Center, Dallas, TX, USA
}

\section{Keywords}

Chronic kidney disease $\cdot$ Monocyte chemoattractant protein-1 - Inflammation - Death · Cardiovascular . Outcomes · Albuminuria · Biomarkers

\footnotetext{
Abstract

Background:Monocyte chemoattractant protein-1 (MCP-1), a marker of inflammation and monocyte recruitment to atherosclerotic plaques, is associated with cardiovascular (CV) outcomes in patients with acute coronary syndrome. Although plasma levels are elevated in chronic kidney disease (CKD), associations with reduced kidney function or outcomes in CKD have not been explored. Methods: In this population-based, probability-sampled, longitudinal cohort of 3,257 participants, including 286 (8.8\%) patients with CKD, we studied the association of plasma MCP-1 with estimated glomerular filtration rate (eGFR), albuminuria, death, and intermediate and hard CV outcomes in CKD and non-CKD individuals. Cox proportional hazards regression
}

assessed associations of baseline MCP-1 with all-cause death and atherosclerotic events. Results: MCP-1 was higher in CKD than non-CKD participants $(p<0.001)$, and negatively associated with eGFR $(r=-0.23, p<0.0001)$ but not albuminuria in CKD. MCP-1 was associated with pulse wave velocity and coronary artery calcification in non-CKD but not CKD individuals. At 13.5 years, there were 230 (7.7\%) deaths and $168(6.4 \%)$ atherosclerotic events in the nonCKD vs. 97 (34.0\%) deaths and 62 (27.9\%) events in the CKD group ( $p<0.001$ for each). MCP-1 was associated with death (hazards ratio [HR] 2.0 [1.4-2.9] per log-unit increase) and atherosclerotic events (1.7 [1.0-2.9]) in CKD individuals. The HR for death in CKD remained significant (1.6 [1.1-2.3]) after adjusting for $\mathrm{CV}$ risk factors. Conclusions: Although plasma MCP-1 increased with decreased eGFR, it remained an independent risk factor for death in CKD. MCP-1 did not correlate with intermediate $\mathrm{CV}$ outcomes, implicating pathways other than atherosclerosis in the association of MCP-1 with death in CKD.

\section{KARGER}

(c) 2018 S. Karger AG, Basel

E-Mail karger@karger.com

www.karger.com/ajn
S. Susan Hedayati, MD, MHSc

Division of Nephrology, Department of Medicine University of Texas Southwestern Medical Center 5939 Harry Hines Blvd., MC 8516, Dallas, TX 75390 (USA)

E-Mail susan.hedayati@utsouthwestern.edu 


\section{Introduction}

Persons with chronic kidney disease (CKD) are at a disproportionately higher risk of cardiovascular (CV) disease and death than non-CKD individuals, and are substantially more likely to die, primarily of $\mathrm{CV}$ disease, than to reach dialysis dependence $[1,2]$. Prognostic models based on traditional CV risk factors do not predict outcomes as accurately in people with CKD vs. those without CKD [3, 4]. This highlights the need to identify non-traditional factors to improve risk prediction in the growing CKD population.

Monocyte chemoattractant protein-1 (MCP-1) is a chemokine produced by macrophages, vascular smooth muscle cells, and endothelial cells in response to hypercholesterolemia and other causes of arterial injury, which is thought to play a role in early monocyte recruitment to atherosclerotic plaques and areas of inflammation $[5,6]$. Plasma MCP-1 levels are higher in advanced CKD than in those with normal kidney function [7]. It is unclear whether increased levels are driven by lower renal clearance or upregulated MCP-1 production in the setting of systemic inflammation associated with CKD [8-10]. Uremic toxins, such as p-cresol and indoxyl sulfate, upregulate MCP-1 production by vascular smooth muscle and endothelial cells in vitro $[11,12]$. Corresponding correlations between these factors and MCP-1 were shown in hemodialysis patients [13], indicating that decreased renal clearance is likely not the only factor underlying MCP-1 elevation in CKD.

Elevated plasma MCP-1 is associated with CV mortality in overweight asymptomatic outpatients and individuals with known coronary artery disease (CAD) or acute coronary syndrome (ACS) [14-17]. However, the few studies that reported associations between MCP-1 and $\mathrm{CV}$ outcomes in CKD or hemodialysis patients were limited by small samples, too few events to allow for adjustment, inclusion of only those with ACS or CAD, or lack of hard CV outcome events $[8,18,19]$.

We sought to test the hypothesis of elevated plasma MCP-1 level being associated with death and CV events using a large, multi-ethnic cohort where both intermediate and hard CV outcomes were measured. The first aim was to investigate associations of plasma MCP-1 with lower estimated glomerular filtration rate (eGFR), increased albuminuria, and intermediate $\mathrm{CV}$ outcomes, such as coronary artery calcification (CAC), left ventricular hypertrophy $(\mathrm{LVH})$, and aortic pulse wave velocity (PWV), in order to explore mechanisms by which MCP-1 may lead to poor outcomes. The second aim was to inves- tigate whether plasma MCP-1 was independently associated with all-cause death or atherosclerotic $\mathrm{CV}$ disease (ASCVD) events and whether CKD presence would modify this association, after controlling for traditional $\mathrm{CV}$ risk factors and eGFR.

\section{Materials and Methods}

\section{Study Participants}

The Dallas Heart Study (DHS) is a community-based multiethnic longitudinal cohort study of residents of Dallas County, Texas [20], approved by the University of Texas Southwestern Institutional Review Board, and adherent to the Declaration of Helsinki. A total of 6,101 persons provided informed consent and completed a visit to collect health-related data. Of those, 3,398 participants provided fasting blood and first-void urine samples, and 2,971 participants underwent advanced imaging studies for measurement of intermediate CV phenotypes. Our primary analysis included 3,257 with measurement of plasma MCP-1 level, urinary albumin-to-creatinine ratio (ACR), and information available for calculation of eGFR (Fig. 1). Participants were followed for a median of 13.5 years.

\section{MCP-1 Measurement}

Fasting venous blood samples were stored in ethylenediaminetetraacetic acid tubes for $\leq 4 \mathrm{~h}$ at $4{ }^{\circ} \mathrm{C}$, then centrifuged at $1,430 \mathrm{~g}$ for $15 \mathrm{~min}$. Plasma was separated and frozen at $-80^{\circ} \mathrm{C}$ until assays were performed in duplicate at Biosite Inc., (now Alere, San Diego, CA, USA) in 384-well microtiter plates on a high-throughput robotic platform (TECAN Genesis RSP 200/8). The binding of alkaline phosphatase-conjugated antibody quantified the plasma concentration of MCP-1, with a reportable range of 40-2,000 pg/mL $[14,19]$.

\section{Urinary and Kidney Function Measurements}

Because the alkaline picrate assay was used to determine serum creatinine concentrations, the 4-variable Modification of Diet in Renal Disease (MDRD) study formula was used to calculate eGFR $[21,22]$. ACR was calculated in $\mathrm{mg} / \mathrm{g}$ from spot urinary albumin and creatinine measured from a first-void urine sample. CKD was defined as either eGFR $<60 \mathrm{~mL} / \mathrm{min} / 1.73 \mathrm{~m}^{2}$ or elevated ACR ( $\geq 17 \mathrm{mg} / \mathrm{g}$ in men or $\geq 25 \mathrm{mg} / \mathrm{g}$ in women) $[23,24]$. CKD was staged as follows: stage 1 , elevated ACR with eGFR $\geq 90$; stage 2, elevated ACR with eGFR 60-89; stage 3, eGFR 30-59; stage 4, eGFR 15-29; and stage 5, eGFR $<15$ [23], not receiving chronic dialysis.

\section{Outcomes}

The pre-specified primary outcome was all-cause death, as ascertained using the National Death Index. The pre-specified secondary outcome was ASCVD events, defined as the composite of myocardial infarction, stroke, coronary revascularization, and CV death. Tertiary outcomes were each component of the secondary outcome and intermediate $\mathrm{CV}$ phenotypes, including CAC, $\mathrm{LVH}$, and PWV. As intermediate CV outcomes were not measured in all participants, these analyses were limited to individuals with available data $(n=2,608$, including 2,396 non-CKD and 212 CKD participants, Fig. 1). CV events were captured by searching hospital 


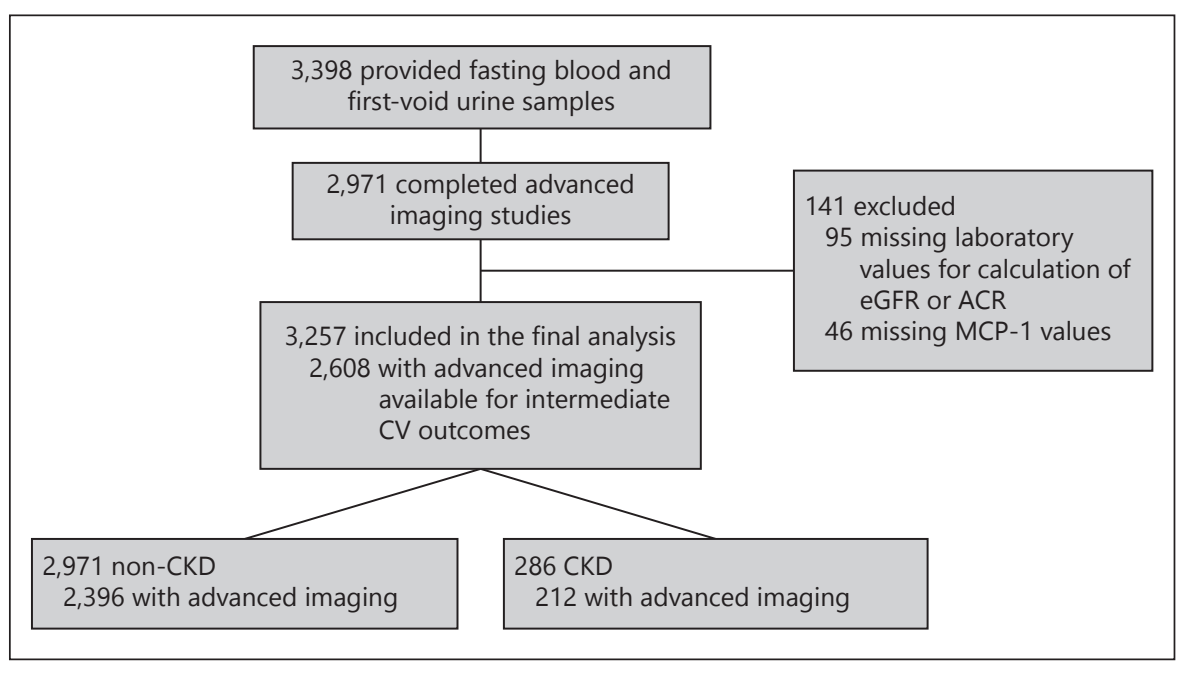

Fig. 1. Flow chart of the study participants.

records, queries, and final adjudication by DHS investigators. Analyses of CV events included 2,831 individuals who had been adjudicated for CV outcomes through 2012. Deaths were considered CV deaths if they were coded with International Statistical Classification of Diseases 10 codes I00-I99 [25].

\section{Intermediate CV Phenotype Measures}

Analyses of intermediate CV phenotypes were conducted for the 2,608 participants who underwent advanced imaging studies. A total of 2,549 participants underwent cardiac computed tomography, 2,575 cardiac magnetic resonance imaging (MRI), and 2,077 aortic MRI. CAC was measured with electron-beam computed tomography on a single scanner (Imatron $150 \mathrm{XP}$, Imatron, Inc., San Francisco, CA, USA) at $80 \%$ of the R-R interval with 30 $\mathrm{cm}$ field of view, 512 matrix with sharp kernel reconstruction [26]. The final score was the mean of 2 consecutive measurements or a single measurement when only one scan was performed [27]. CAC $\geq 100$ Agatston units was pre-specified as clinically relevant, which corresponds to moderate to high 10-year CV event risk [28].

MRI was performed with a Phillips Medical Systems (Best, The Netherlands) 1.5-Tesla Intera magnet. LV mass was measured in $\mathrm{g} / \mathrm{m}^{2}$ indexed to body surface area (BSA) [29]. LVH was defined as LV mass/BSA $>97.5$ th sex-based percentile, which was $>89 \mathrm{~g} / \mathrm{m}^{2}$ in men and $>112 \mathrm{~g} / \mathrm{m}^{2}$ in women in a healthy subpopulation of the DHS [29]. The ascending and descending thoracic aorta were imaged using prospective electrocardiogram gating and a breath-hold, velocity-encoded, phase-contrast gradient echo sequence as previously described [30, 31]. Aortic PWV was calculated in $\mathrm{m} / \mathrm{s}$ by dividing arch distance by transit time, with higher velocity indicative of a stiffer aorta. Transit time was calculated as the time difference between ascending and descending upstroke velocities at half maximum [30].

\section{Statistical Analysis}

The analysis was stratified into $\mathrm{CKD}$ and non-CKD groups to address the question of whether CKD presence modifies the association of MCP-1 with intermediary and hard CV outcomes. Clinical variables and outcome measures were compared between individuals with no CKD, stages 1-2 CKD, and stages 3-5 CKD. $P$ values for trends were derived using Cochran-Armitage tests for categorical and Jonckheere-Terpstra tests for continuous vari- ables. Correlations of MCP-1 with eGFR and ACR were tested using Spearman correlations. Continuous variables with a nonGaussian distribution (plasma MCP-1, eGFR, ACR, aortic PWV, and LV mass/BSA) were log-transformed prior to comparisons.

Time to event was estimated using Kaplan-Meier survival analysis and compared among quartiles of MCP-1 using log-rank tests. Associations of log-transformed MCP-1 with each outcome were determined using Cox proportional hazards regression. Models controlled for traditional CV risk factors: age, sex, race, diabetes mellitus, hypertension, current smoking, total and high-density lipoprotein cholesterol. Additional models also controlled for eGFR to account for the effect of renal clearance on plasma MCP-1 levels, and high sensitivity C-reactive protein (hsCRP) to account for generalized inflammation. Effect modification of CKD was also tested and considered statistically significant if the CKD-MCP-1 interaction $p$ value was $<0.1$. Sensitivity analyses of those without prior CV disease (self-reported history of myocardial infarction, revascularization, heart failure, or stroke) included 3,017 participants (236 with CKD and 2,781 without CKD). Statistical analyses were performed with SAS 9.4 and SAS Enterprise Guide 7.1 (SAS Institute, Cary, NC, USA).

\section{Results}

\section{Baseline Characteristics}

There were no significant differences between participants who were excluded due to missing MCP-1 values as compared to those who were included, except for a higher proportion of smokers (online suppl. Table 1; for all online suppl. material, see www.karger.com/ doi/10.1159/000488806). Of the 3,257 participants included, 56\% were female, 52\% Black, 29\% Caucasian, $17 \%$ Hispanic, and $2 \%$ of other races. The total number with CKD was $286(8.8 \%)$. There were 217 participants with CKD stages $1-2$ and 69 with stages $3-5$. There were significant trends in the prevalence of most traditional $\mathrm{CV}$ 
Table 1. Baseline characteristics of the cohort by CKD status

\begin{tabular}{|c|c|c|c|c|}
\hline Variable & $\begin{array}{l}\text { Non-CKD } \\
(n=2,971)\end{array}$ & $\begin{array}{l}\text { CKD stages } 1-2 \\
(n=217)\end{array}$ & $\begin{array}{l}\text { CKD stages } 3-5 \\
(n=69)\end{array}$ & $\begin{array}{l}p \text { value } \\
\text { for trend }\end{array}$ \\
\hline Age, years, median (IQR) & $43.0(36.0-51.0)$ & $47.0(38.0-54.0)$ & $53.0(47.0-58.0)$ & $<0.0001$ \\
\hline Race, $n(\%)$ & & & & $<0.0001^{\mathrm{a}}$ \\
\hline Black & $1,503(50.6)$ & $151(69.6)$ & $44(63.7)$ & \\
\hline White & $889(29.9)$ & $28(12.9)$ & $19(27.5)$ & \\
\hline Other & $63(2.1)$ & $2(0.9)$ & $3(4.4)$ & \\
\hline Current smoker, $n(\%)$ & $1,323(44.6)$ & $112(51.6)$ & $50(58.0)$ & 0.004 \\
\hline Hypertension, $n(\%)$ & $985(33.2)$ & $134(61.8)$ & $56(81.2)$ & $<0.0001$ \\
\hline Diabetes mellitus, $n(\%)$ & $283(9.5)$ & $84(38.7)$ & $22(31.9)$ & $<0.0001$ \\
\hline Hyperlipidemia, $n(\%)$ & $376(12.7)$ & $30(13.9)$ & $15(21.7)$ & 0.05 \\
\hline Prior CV disease, $n(\%)^{\mathrm{b}}$ & $190(6.4)$ & $30(13.8)$ & $20(29.0)$ & $<0.0001$ \\
\hline Total cholesterol, mg/dL, median (IQR) & $177.0(154.0-203.0)$ & $174.0(150.0-202.0)$ & $182.0(157.0-196.0)$ & 0.60 \\
\hline HDL cholesterol, mg/dL, median (IQR) & $47.0(40.0-57.0)$ & $47.0(39.0-54.0)$ & $47.0(38.0-59.0)$ & 0.29 \\
\hline ACR, mg/g, median (IQR) & $2.7(1.8-4.6)$ & $48.7(29.3-107.8)$ & $16.2(3.2-105.3)$ & $<0.0001$ \\
\hline $\mathrm{ACR}, \mathrm{mg} / \mathrm{g}$, mean $(\mathrm{SD})$ & $3.9(3.6)$ & $172.4(426.1)$ & $308.9(993.4)$ & $<0.0001$ \\
\hline $\mathrm{eGFR}, \mathrm{mL} / \mathrm{min} / 1.73 \mathrm{~m}^{2}$, median (IQR) & $98.4(85.8-113.2)$ & $99.8(83.1-117.5)$ & $49.1(38.2-55.9)$ & $<0.0001$ \\
\hline MCP-1, pg/mL, median (IQR) & $164.7(120.2-221.0)$ & $183.0(138.9-246.0)$ & $233.8(177.5-325.8)$ & $<0.0001$ \\
\hline
\end{tabular}

${ }^{a}$ Represents $p$ value for Chi-square test.

${ }^{b}$ Prior CV disease was defined as self-reported history of prior myocardial infarction, revascularization, heart failure, or stroke.

ACR, urine albumin-to-creatinine ratio; BMI, body mass index; CKD, chronic kidney disease; CV, cardiovascular; eGFR, estimated glomerular filtration rate; HDL, high density lipoprotein; IQR, interquartile range; MCP-1, monocyte chemoattractant protein-1.

risk factors across the 3 groups, including smoking, hypertension, diabetes, and prior CV disease (Table 1), with a higher prevalence among those with CKD stages 1-2 than no CKD, and among those with CKD stages 3-5 as compared with stages 1-2. Age also increased across groups.

\section{Correlations of Plasma MCP-1 with Kidney Function and Albuminuria}

MCP-1 levels were higher in CKD than in non-CKD participants (median [interquartile range] 192.2 [143.6$269.8]$ vs. 164.7 [120.3-220.9] pg/mL, $p<0.001)$, and increased with lower eGFR and across advancing CKD stages, $p<0.001$ (Table 1, Fig. 2). In both the CKD and non-CKD groups, eGFR was lower across higher MCP-1 quartiles, but no association was seen with ACR (online suppl. Tables 2,3). In the entire cohort, a similar relationship between eGFR and MCP-1 quartiles was seen, and there was a difference in ACR between the first quartile of MCP-1 and the third and fourth quartiles (online suppl. Tables 2, 3). MCP-1 correlated negatively with eGFR,
Spearman rho -0.12 in the entire cohort, -0.10 in non$\mathrm{CKD}$, and -0.23 in $\mathrm{CKD}$, which remained significant after adjusting for age (online suppl. Table $4, p<0.001$ for all correlations). There was also a modest but significant association between albuminuria and MCP-1 in the entire cohort ( rho $=0.07, p<0.0001$ ), but no association was seen in those with CKD (CKD rho $=-0.02, p=0.68$, online suppl. Table 4).

\section{Associations of MCP-1 with Intermediate CV Phenotypes}

Participants with CKD exhibited higher CAC, PWV, LV mass/BSA, and LVH prevalence compared to those without CKD (Table 2). PWV, LV mass/BSA, and the prevalence of $\mathrm{CAC} \geq 100$ were higher with more advanced CKD stages, but there was no difference in the proportion with $\mathrm{LVH}$ (Table 2). In the non-CKD group, the prevalence of CAC $\geq 100$ increased from $4.8 \%$ in the first MCP- 1 quartile to $10.9 \%$ in the fourth quartile ( $p<0.001$ for trend, Table 3 ). There were also associations between higher MCP-1 quartiles and higher aortic PWV ( $p=0.001$ for trend), but not 

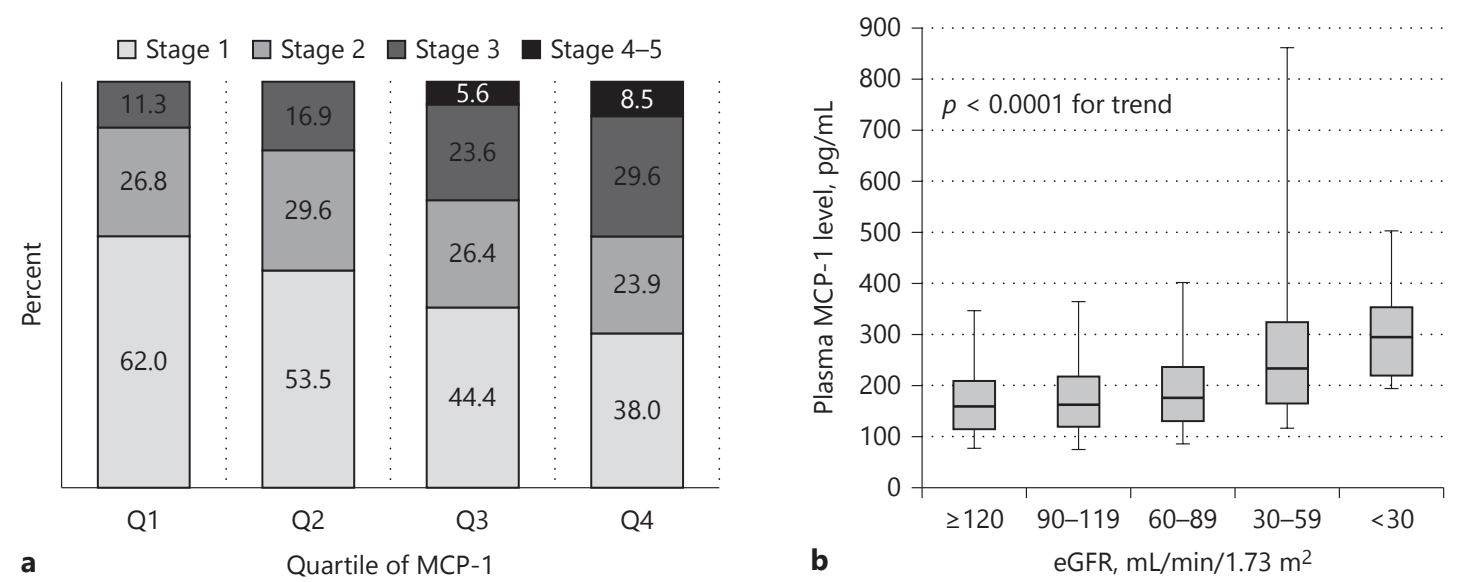

Fig. 2. Relationship between plasma MCP-1 and kidney function. a Distribution of stages of CKD by quartile of plasma MCP-1. b Median plasma MCP-1 levels by eGFR. Boxes represent the median values and IQR. Error bars represent the 5 and 95th percen-

tiles. CKD, chronic kidney disease; eGFR, estimated glomerular filtration rate; $\mathrm{MCP}-1$, monocyte chemoattractant protein-1; IQR, interquartile ranges.

Table 2. Intermediate CV Outcomes by CKD status

\begin{tabular}{|c|c|c|c|c|c|c|}
\hline Variable & $\begin{array}{l}\text { Non-CKD } \\
(n=2,396)\end{array}$ & $\begin{array}{l}\text { Any CKD } \\
(n=212)\end{array}$ & $p$ value & $\begin{array}{l}\text { CKD 1-2 } \\
(n=165)\end{array}$ & $\begin{array}{l}\text { CKD 3-5 } \\
(n=47)\end{array}$ & $p$ value \\
\hline CAC $\geq 100$ Agatston units, $n(\%)$ & $200(8.4)$ & $46(21.8)$ & & $27(16.6)$ & $18(40.0)$ & 0.0007 \\
\hline LV mass/BSA, g/m², median (IQR) & $79.6(69.8-92.1)$ & $91.9(78.5-110.9)$ & $<0.001$ & $93.2(81.1-112.1)$ & $83.6(70.9-101.9)$ & 0.02 \\
\hline $\mathrm{LVH}, n(\%)$ & $225(9.4)$ & $69(32.6)$ & $<0.001$ & $57(34.6)$ & 12.(26.7) & 0.32 \\
\hline Aortic PWV, m/s, median (IQR) & $4.2(3.3-5.6)$ & $5.4(4.0-7.0)$ & $<0.001$ & $5.3(3.9-6.7)$ & $6.6(4.4-8.6)$ & 0.03 \\
\hline
\end{tabular}

Includes 2,608 participants who underwent testing for intermediate CV outcomes.

BSA, body surface area; CAC, coronary artery calcification; CKD, chronic kidney disease; IQR, interquartile range; LV, left ventricular; LVH, left ventricular hypertrophy; PWV, pulse wave velocity.

with LV mass/BSA or LVH in the non-CKD group. In the CKD group, no associations were observed between MCP-1 quartiles and intermediate CV phenotypes (Table 3).

\section{Association of MCP-1 with Death and CV Outcomes}

There were 327 deaths and 230 ASCVD events over median (interquartile range) follow-up of 161.5 (157.9166.7) months. Events occurred with a higher frequency in CKD than non-CKD individuals $(p<0.001$ for each event, Fig. 3). Higher MCP-1 quartiles were associated with a higher proportion of deaths ( $p$ for trend $=0.001$ in $\mathrm{CKD},<0.001$ in non-CKD), but no difference was observed for ASCVD events (Table 3). In the CKD group, there was a significant trend toward increased $\mathrm{CV}$ death across MCP-1 quartiles (Table 3). MCP-1, taken continuously, was associated with time to all-cause death in the entire cohort and in the CKD and non-CKD subgroups (Fig. 3). Higher MCP-1 quartiles were associated with death in both CKD $(\log -$ rank $p=0.0004)$ and non-CKD $(p<0.001)$ groups (Fig. 4).

Adjusting for traditional CV risk factors attenuated the magnitude of these associations, but MCP-1 remained independently associated with death in both groups (CKD group, adjusted hazards ratio $=1.6,95 \%$ CI $1.1-$ 2.3). Adjustments for eGFR and hsCRP further attenuated the association in the CKD group, but MCP-1 remained associated with death in both groups (all CKDMCP-1 interaction $p$ values $>0.1$; Table 4 ). Sensitivity analyses including 3,017 individuals without prior CV disease revealed similar results (Table 4).

In unadjusted models, MCP-1 was associated with ASCVD events in the entire cohort and the CKD group, 
Table 3. Outcomes by quartile of MCP-1 and CKD status

\begin{tabular}{|c|c|c|c|c|}
\hline Variable & \multicolumn{4}{|c|}{ Non-CKD $(n=2,971)$, quartile of MCP-1 (range, pg/mL) } \\
\hline \multicolumn{5}{|l|}{ Intermediate outcomes } \\
\hline CAC $\geq 100$ Agatston units, $n(\%)$ & $27(4.8)$ & $53(8.8)$ & $53(9.1)$ & $65(10.9)^{\mathrm{a}}$ \\
\hline LV mass/BSA, $\mathrm{g} / \mathrm{m}^{2}$, median (IQR) & $79.0(69.8-91.3)$ & $79.8(69.8-92.2)$ & $79.7(69.6-92.0)$ & $79.7(70.1-92.8)$ \\
\hline \multicolumn{5}{|l|}{ Hard outcomes $n(\%)$} \\
\hline All-cause death & $33(5.1)$ & $57(8.4)$ & $53(8.0)$ & $87(12.8)^{\mathrm{a}}$ \\
\hline ASCVD events & $43(6.7)$ & $36(5.5)$ & $36(5.7)$ & $51(7.9)$ \\
\hline Myocardial infarction & $14(2.2)$ & $12(1.8)$ & $13(2.0)$ & $20(3.1)$ \\
\hline Stroke & $14(2.2)$ & $8(1.2)$ & $13(2.0)$ & $18(2.8)$ \\
\hline $\mathrm{CV}$ revascularization & $20(3.1)$ & $21(3.2)$ & $23(3.6)$ & $27(4.2)$ \\
\hline Variable & $\begin{array}{l}\text { Q1 (48.9-142.9) } \\
(n=71)\end{array}$ & $\begin{array}{l}\text { Q2 (143.0-191.9) } \\
(n=72)\end{array}$ & $\begin{array}{l}\text { Q3 (192.0-270.9) } \\
(n=72)\end{array}$ & $\begin{array}{l}\text { Q4 (271.0-2,004.0) } \\
(n=71)\end{array}$ \\
\hline \multicolumn{5}{|l|}{ Intermediate outcomes } \\
\hline CAC $\geq 100$ Agatston units, $n(\%)$ & $8(14.8)$ & $12(25.5)$ & $10(17.9)$ & $15(29.4)$ \\
\hline LV mass/BSA, $\mathrm{g} / \mathrm{m}^{2}$, median (IQR) & $96.0(84.7-108.1)$ & $87.8(77.6-106.6)$ & $86.1(74.8-107.6)$ & $93.4(83.7-126.5)$ \\
\hline $\mathrm{LVH}, n(\%)$ & $16(32.0)$ & $15(28.3)$ & $16(28.6)$ & $22(43.1)$ \\
\hline Aortic PWV, m/s, median (IQR) & $5.4(4.0-6.7)$ & $5.5(3.9-6.8)$ & $5.1(3.6-7.0)$ & $5.6(4.5-8.4)$ \\
\hline \multicolumn{5}{|l|}{ Hard outcomes, $n(\%)$} \\
\hline All-cause death & $15(24.2)$ & $24(35.8)$ & $23(34.3)$ & $35(53.8)^{\mathrm{a}}$ \\
\hline ASCVD events & $14(25.5)$ & $13(22.8)$ & $17(29.3)$ & $18(35.3)$ \\
\hline Myocardial infarction & $5(9.1)$ & $2(3.5)$ & $6(10.3)$ & $4(7.8)$ \\
\hline
\end{tabular}

${ }^{\mathrm{a}} p<0.05$ for trend.

ASCVD, atherosclerotic cardiovascular disease; BSA, body surface area; CAC, coronary artery calcification; CKD, chronic kidney disease; CV, cardiovascular; IQR, interquartile range; LV, left ventricular; LVH, left ventricular hypertrophy; MCP-1, monocyte chemoattractant protein-1.

but not in the non-CKD group (Fig. 3). These associations became non-significant after adjusting for $\mathrm{CV}$ risk factors (Table 4). There were no significant CKD-MCP-1 interactions for ASCVD events. Sensitivity analysis in individuals without prior CV disease yielded similar results (Table 4).

\section{Discussion}

To our knowledge, this is the first study that reports the association of plasma MCP-1 with intermediate and hard $\mathrm{CV}$ outcomes using a large, multi-ethnic, longitudinal co- hort based on the presence of CKD. Importantly, we show that plasma MCP-1 levels (1) are higher with lower eGFR and across higher CKD stages but do not correlate with albuminuria in CKD patients; (2) independently associate with death after adjusting for traditional CV risk factors in both CKD and non-CKD individuals; and (3) do not independently associate with intermediate $\mathrm{CV}$ phenotypes or ASCVD events in CKD individuals. This suggests that the association observed with death may be mediated through pathways other than atherosclerosis. Given that many other known CV risk factors perform less well in CKD than non-CKD individuals, it is notable that MCP-1 predicts death equally as well in CKD as in non-CKD individuals. 
Fig. 3. Associations of log-transformed MCP-1 with outcomes by CKD status. Forest plot depicts the unadjusted hazards ratios and 95\% CI for outcomes stratified by CKD status. ASCVD, atherosclerotic cardiovascular disease; $\mathrm{CKD}$, chronic kidney disease; CV, cardiovascular; MCP-1, monocyte chemoattractant protein-1.

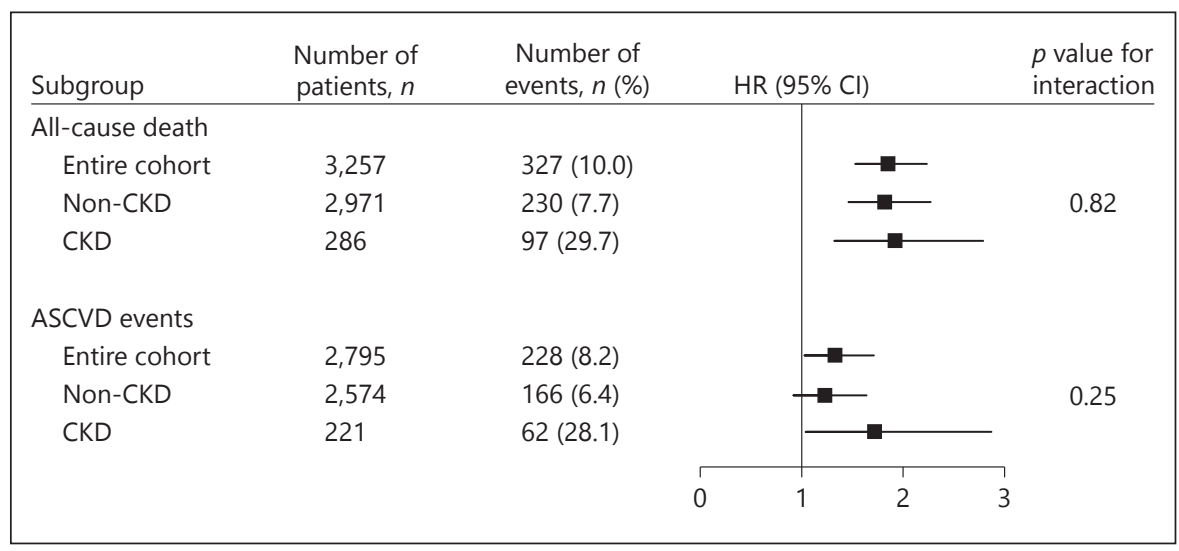

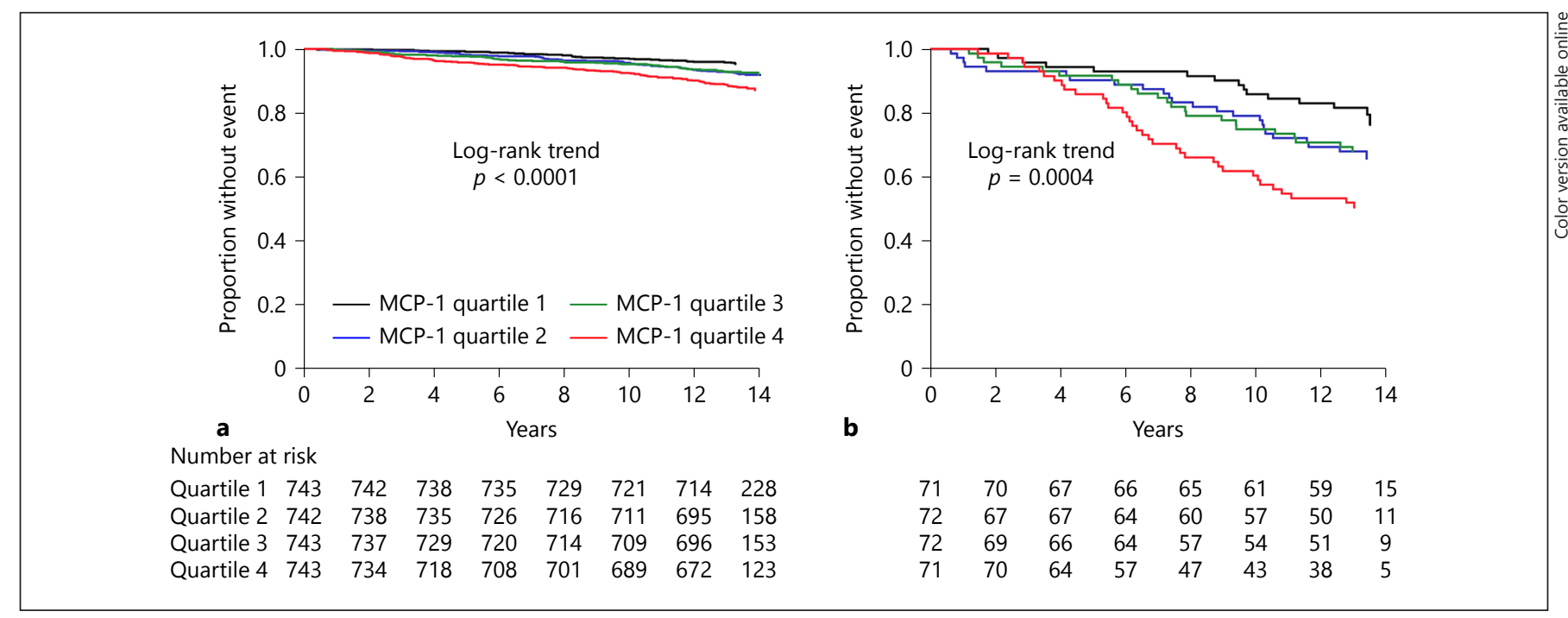

Fig. 4. Association of MCP-1 quartiles with all-cause death by CKD status. Kaplan-Meier curves depict the association of quartiles of MCP-1 with all-cause death in (a) non-CKD and (b) CKD groups. CKD, chronic kidney disease, MCP-1, monocyte chemoattractant protein-1.
Prior studies from the DHS reported that MCP-1 was highly correlated with age [19], which is included in the calculation of eGFR. In our study, MCP-1 remained correlated with eGFR after adjusting for age, suggesting an independent relationship between decreased kidney function and MCP-1. The only other published study demonstrating an association between MCP-1 and eGFR used data from the African American Diabetes Heart Study [10]. Because black individuals differ in patterns of both kidney and CV disease than non-blacks, it is important that we demonstrated this finding in a large racially and ethnically diverse sample. Possible explanations include decreased renal clearance of MCP-1, generalized inflammation associated with $\mathrm{CKD}$, or upregulation of
MCP-1 production in the setting of uremia $[9,32]$. In this study, only $5 \%$ of the variability in MCP-1 was accounted for by eGFR in those with CKD, suggesting that additional underlying processes in individuals with CKD that either increase MCP-1 production or decrease its breakdown may lead to elevated levels. It is possible that generalized inflammation in CKD at least partially drives increased plasma MCP-1 levels [6, 9].

Although there was a modest but statistically significant association between MCP-1 and albuminuria in the entire cohort, this association became non-significant when stratifying by CKD presence. It is possible that an association between albuminuria and MCP-1 may not have been apparent in our cohort comprised largely of 
CV events, and the study was likely underpowered to detect a difference. To our knowledge, no other published studies investigated the association of plasma MCP-1 with outcomes in CKD participants, despite evidence suggesting a role for MCP-1 in atherogenesis in the general population, and data suggesting an association of MCP-1 with kidney function decline.

The correlation of MCP-1 with lower eGFR implies that decreased renal clearance may account for some of the association of MCP-1 with outcomes. Indeed, adjusting for eGFR slightly attenuated the association of MCP-1 with all-cause death. Another contributing factor may be the presence of systemic inflammation in CKD, previously implicated in oxidative stress, endothelial dysfunction, atherosclerosis, malnutrition, and CKD progression [3739], reflected by the attenuation of the association of MCP-1 with death when adjusting for hsCRP. Monocyte chemotaxis mediated through MCP-1 may account for an important element of the inflammation-associated mortality risk in CKD.

We also sought to characterize the mechanisms by which MCP-1 may lead to adverse outcomes by exploring associations of MCP-1 with CV disease intermediaries. While higher MCP-1 levels were associated with elevated CAC and PWV in non-CKD participants, no such correlations were observed in CKD individuals. However, elevated CAC and PWV were more prevalent in CKD vs. nonCKD participants, even in a sample heavily weighted toward earlier CKD stages. These findings may suggest that although MCP-1 is involved in the development of atherosclerosis in the general population, the worsening of these intermediate CV phenotypes in CKD may depend on other pathogenic mechanisms. It is known that individuals with CKD develop medial arterial calcification in addition to intimal atherosclerotic calcified plaques as seen in the general population [40], which cannot be distinguished using CAC. It is not clear whether this results from a more severe manifestation of the same underlying processes, or whether CKD upregulates different pathogenic pathways to cause this medial calcification [40]. The absence of association between MCP-1 and elevated CAC in CKD participants could suggest that MCP-1 may lead to death through mechanisms other than calcific atherosclerosis. Although there was a trend seen toward increased CV deaths across MCP-1 quartiles in the CKD group, the number of outcome events was too low to derive definitive conclusions. Alternatively, the association seen between MCP-1 and death but not ASCVD events in adjusted models may implicate traditional CV risk factors in the excess ASCVD risk, or that non-atherosclerotic disease pathways, such as infection or cancer, link monocyte chemotaxis to all-cause death in CKD individuals. We did not have non-CV causes of death available to further explore this hypothesis.

Several limitations deserve mentioning. First, the effect of time-varying measures of plasma MCP-1 on outcomes was not assessed. Given the separation in mortality between MCP-1 quartiles occurred approximately $2-3$ years after baseline MCP-1 was measured, the predictive value of repeated measures of MCP-1 deserves exploration. Second, since creatinine was measured using the alkaline picrate assay, we were unable to use the CKD-EPI equation, which estimates GFR more accurately than the MDRD equation for eGFR $>60$ [41]. However, a sensitivity analysis using the CKD-EPI equation showed that only 3 participants were misclassified by using the MDRD equation. Third, the cohort had relatively few persons with CKD stages 4-5, limiting generalizability to this segment of CKD. However, about $75 \%$ of the cohort was comprised of early CKD stages with preserved eGFR, where interventions would make the highest impact. Fourth, not all participants underwent imaging for intermediate $\mathrm{CV}$ outcomes, slightly decreasing the sample size for these analyses. Finally, power may have limited the finding of an independent association between MCP-1 and ASCVD events in CKD participants. However, the fact that a similar association was not found in the larger non-CKD group comprised of about 3,000 individuals makes the lack of power a less likely explanation.

In conclusion, plasma MCP-1 was associated with decreased eGFR in this multi-ethnic community-based cohort. Despite this, MCP-1 was independently associated with death in both CKD and non-CKD participants, even after controlling for traditional CV risk factors. MCP-1 may mediate mortality through pathways involving inflammation. MCP-1 was also associated with ASCVD events in unadjusted models, suggesting that atherosclerotic events likely contribute to but do not fully account for the association of MCP-1 with mortality. Despite worse $\mathrm{CV}$ outcomes in those with $\mathrm{CKD}$, associations between MCP-1 and CV disease intermediaries were nonsignificant in CKD individuals, further implicating pathways other than atherosclerosis in the association of MCP-1 with death in CKD. Future studies should investigate whether pathways that activate MCP-1 are altered in the uremic milieu and identify other circulating biomarkers that may clarify the pathogenesis of CV disease in CKD. The clinical utility of plasma MCP-1 for mortality prognostication should be validated in larger cohorts with advanced CKD. 


\section{Acknowledgments}

Parts of these data were presented in abstract form on April 19, 2017, at the 2017 Spring Clinical Meeting of the National Kidney Foundation in Orlando, FL, and on November 4, 2017, at the American Society of Nephrology Kidney Week Meeting in New Orleans, LA.

\section{Financial Disclosure}

J.A. de Lemos has received grant support and consulting income from Roche Diagnostics and Abbott Diagnostics and has served on endpoint committees for Siemen's Health Care Diagnostics and Radiometer.

\section{Funding}

The DHS was supported by a grant from the Donald W. Reynolds Foundation and by USPHS GCRC grant No. M01-RR00633 from NIH/NCRR-CR. This work was supported in part by UT Southwestern O'Brien Kidney Research Core Center (NIDDK, P30DK079328). Research reported in this publication was also supported by the National Center for Advancing Translational Sciences of the National Institutes of Health under award number UL1TR001105 to the University of Texas Southwestern Medical Center, as well as grant T32DK007257 from the National Institute of Diabetes and Digestive and Kidney Diseases, National Institutes of Health (L.P. Gregg).

The content is solely the responsibility of the authors and does not necessarily represent the official views of the National Institutes of Health or the Department of Veterans Affairs.

\section{References}

-1 Go AS, Chertow GM, Fan D, McCulloch CE, Hsu CY: Chronic kidney disease and the risks of death, cardiovascular events, and hospitalization. N Engl J Med 2004;351:1296-1305.

-2 Dalrymple LS, Katz R, Kestenbaum B, Shlipak MG, Sarnak MJ, Stehman-Breen C, Seliger S, Siscovick D, Newman AB, Fried L: Chronic kidney disease and the risk of end-stage renal disease versus death. J Gen Intern Med 2011; 26:379-385

-3 Sarnak MJ, Levey AS, Schoolwerth AC, Coresh J, Culleton B, Hamm LL, McCullough PA, Kasiske BL, Kelepouris E, Klag MJ, Parfrey P, Pfeffer M, Raij L, Spinosa DJ, Wilson PW; American Heart Association Councils on Kidney in Cardiovascular Disease, High Blood Pressure Research, Clinical Cardiology, and Epidemiology and Prevention: Kidney disease as a risk factor for development of cardiovascular disease: a statement from the american heart association councils on kidney in cardiovascular disease, high blood pressure research, clinical cardiology, and epidemiology and prevention. Circulation 2003; 108:2154-2169.

4 Gregg LP, Adams-Huet B, Li X, Colbert G, Jain N, de Lemos JA, Hedayati SS: Effect modification of chronic kidney disease on the association of circulating and imaging cardiac biomarkers with outcomes. J Am Heart Assoc 2017;6:e005235.

5 Gosling J, Slaymaker S, Gu L, Tseng S, Zlot $\mathrm{CH}$, Young SG, Rollins BJ, Charo IF: Mcp-1 deficiency reduces susceptibility to atherosclerosis in mice that overexpress human apolipoprotein B. J Clin Invest 1999;103:773-778.

-6 Jiang Y, Beller DI, Frendl G, Graves DT: Monocyte chemoattractant protein-1 regulates adhesion molecule expression and cytokine production in human monocytes. J Immunol 1992;148:2423-2428.

7 Fukami A, Yamagishi S, Adachi H, Matsui T, Yoshikawa K, Ogata K, Kasahara A, Tsukaga- wa E, Yokoi K, Imaizumi T: High white blood cell count and low estimated glomerular filtration rate are independently associated with serum level of monocyte chemoattractant protein-1 in a general population. Clin Cardiol 2011;34:189-194.

8 Papayianni A, Alexopoulos E, Giamalis P, Gionanlis L, Belechri AM, Koukoudis P, Memmos D: Circulating levels of icam-1, vcam-1, and mcp-1 are increased in haemodialysis patients: association with inflammation, dyslipidaemia, and vascular events. Nephrol Dial Transplant 2002;17:435-441.

-9 Stinghen AE, Goncalves SM, Martines EG, Nakao LS, Riella MC, Aita CA, Pecoits-Filho $\mathrm{R}$ : Increased plasma and endothelial cell expression of chemokines and adhesion molecules in chronic kidney disease. Nephron Clin Pract 2009;111:c117-c126.

10 Murea M, Register TC, Divers J, Bowden DW, Carr JJ, Hightower CR, Xu J, Smith SC, Hruska KA, Langefeld CD, Freedman BI: Relationships between serum mcp-1 and subclinical kidney disease: African american-diabetes heart study. BMC Nephrol 2012;13:148.

-11 Maciel RA, Rempel LC, Bosquetti B, Finco $A B$, Pecoits-Filho R, Souza WM, Stinghen AE: P-cresol but not $\mathrm{p}$-cresyl sulfate stimulate mcp-1 production via NF- $\kappa B$ p65 in human vascular smooth muscle cells. J Bras Nefrol 2016;38:153-160.

12 Masai N, Tatebe J, Yoshino G, Morita T: Indoxyl sulfate stimulates monocyte chemoattractant protein-1 expression in human umbilical vein endothelial cells by inducing oxidative stress through activation of the NADPH oxidase-nuclear factor- $\kappa B$ pathway. Circ J 2010;74:2216-2224.

-13 Borges NA, Barros AF, Nakao LS, Dolenga CJ, Fouque D, Mafra D: Protein-bound uremic toxins from gut microbiota and inflammatory markers in chronic kidney disease. J Ren Nutr 2016;26:396-400.
14 de Lemos JA, Morrow DA, Sabatine MS, Murphy SA, Gibson CM, Antman EM, McCabe $\mathrm{CH}$, Cannon CP, Braunwald E: Association between plasma levels of monocyte chemoattractant protein-1 and long-term clinical outcomes in patients with acute coronary syndromes. Circulation 2003;107:690-695.

15 de Lemos JA, Morrow DA, Blazing MA, Jarolim P, Wiviott SD, Sabatine MS, Califf RM, Braunwald E: Serial measurement of monocyte chemoattractant protein-1 after acute coronary syndromes: Results from the a to Z trial. J Am Coll Cardiol 2007;50:21172124.

16 Ding D, Su D, Li X, Li Z, Wang Y, Qiu J, Lin P, Zhang Y, Guo P, Xia M, Li D, Yang Y, Hu G, Ling W: Serum levels of monocyte chemoattractant protein- 1 and all-cause and cardiovascular mortality among patients with coronary artery disease. PLoS One 2015; 10 : e0120633.

17 Piemonti L, Calori G, Lattuada G, Mercalli A, Ragogna F, Garancini MP, Ruotolo G, Luzi L, Perseghin G: Association between plasma monocyte chemoattractant protein-1 concentration and cardiovascular disease mortality in middle-aged diabetic and nondiabetic individuals. Diabetes Care 2009;32:21052110.

18 Kusano KF, Nakamura K, Kusano H, Nishii N, Banba K, Ikeda T, Hashimoto K, Yamamoto M, Fujio H, Miura A, Ohta K, Morita H, Saito H, Emori T, Nakamura Y, Kusano I, Ohe T: Significance of the level of monocyte chemoattractant protein-1 in human atherosclerosis. Circ J 2004;68:671-676.

19 Deo R, Khera A, McGuire DK, Murphy SA, Meo Neto Jde P, Morrow DA, de Lemos JA: Association among plasma levels of monocyte chemoattractant protein-1, traditional cardiovascular risk factors, and subclinical atherosclerosis. J Am Coll Cardiol 2004;44: 1812-1818. 
-20 Victor RG, Haley RW, Willett DL, Peshock RM, Vaeth PC, Leonard D, Basit M, Cooper RS, Iannacchione VG, Visscher WA, Staab JM, Hobbs HH; Dallas Heart Study I: The dallas heart study: A population-based probability sample for the multidisciplinary study of ethnic differences in cardiovascular health. Am J Cardiol 2004;93:1473-1480.

-21 Kramer H, Toto R, Peshock R, Cooper R, Victor R: Association between chronic kidney disease and coronary artery calcification: the dallas heart study. J Am Soc Nephrol 2005;16: 507-513.

-22 Levey AS, Bosch JP, Lewis JB, Greene T, Rogers N, Roth D: A more accurate method to estimate glomerular filtration rate from serum creatinine: a new prediction equation. Modification of diet in renal disease study group. Ann Intern Med 1999;130: 461-470.

23 National Kidney Foundation: K/DOQI clinical practice guidelines for chronic kidney disease: Evaluation, classification, and stratification. Am J Kidney Dis 2002(2 suppl 1);39:S1S266.

-24 Warram JH, Gearin G, Laffel L, Krolewski AS: Effect of duration of type i diabetes on the prevalence of stages of diabetic nephropathy defined by urinary albumin/creatinine ratio. J Am Soc Nephrol 1996;7:930-937.

-25 Lloyd-Jones D, Adams RJ, Brown TM, Carnethon M, Dai S, De Simone G, Ferguson TB, Ford E, Furie K, Gillespie C, Go A, Greenlund K, Haase N, Hailpern S, Ho PM, Howard V, Kissela B, Kittner S, Lackland D, Lisabeth L, Marelli A, McDermott MM, Meigs J, Mozaffarian D, Mussolino M, Nichol G, Roger VL, Rosamond W, Sacco R, Sorlie P, Stafford R, Thom T, Wasserthiel-Smoller S, Wong ND, Wylie-Rosett J; American Heart Association Statistics C, Stroke Statistics S: Executive summary: heart disease and stroke statistics - 2010 update: a report from the american heart association. Circulation 2010;121:948954.

-26 Agatston AS, Janowitz WR, Hildner FJ, Zusmer NR, Viamonte M Jr, Detrano R: Quantification of coronary artery calcium using ultrafast computed tomography. J Am Coll Cardiol 1990;15:827-832.

-27 Bild DE, Bluemke DA, Burke GL, Detrano R, Diez Roux AV, Folsom AR, Greenland P, Ja- cob DR Jr, Kronmal R, Liu K, Nelson JC, O'Leary D, Saad MF, Shea S, Szklo M, Tracy RP: Multi-ethnic study of atherosclerosis: Objectives and design. Am J Epidemiol 2002;156: 871-881.

28 Baber U, de Lemos JA, Khera A, McGuire DK, Omland T, Toto RD, Hedayati SS: Non-traditional risk factors predict coronary calcification in chronic kidney disease in a population-based cohort. Kidney Int 2008;73:615621.

29 Drazner MH, Dries DL, Peshock RM, Cooper RS, Klassen C, Kazi F, Willett D, Victor RG: Left ventricular hypertrophy is more prevalent in blacks than whites in the general population: the dallas heart study. Hypertension 2005;46:124-129.

30 Maroules CD, Khera A, Ayers C, Goel A, Peshock RM, Abbara S, King KS: Cardiovascular outcome associations among cardiovascular magnetic resonance measures of arterial stiffness: the dallas heart study. J Cardiovasc Magn Reson 2014;16:33.

31 Goel A, Maroules CD, Mitchell GF, Peshock R, Ayers C, McColl R, Vongpatanasin W, King KS: Ethnic difference in proximal aortic stiffness: An observation from the dallas heart study. JACC Cardiovasc Imaging 2017; 10:5461.

-32 Stenvinkel P, Lindholm B, Heimburger M, Heimburger O: Elevated serum levels of soluble adhesion molecules predict death in predialysis patients: association with malnutrition, inflammation, and cardiovascular disease. Nephrol Dial Transplant 2000;15: 1624-1630.

33 Chiarelli F, Cipollone F, Mohn A, Marini M, Iezzi A, Fazia M, Tumini S, De Cesare D, Pomilio M, Pierdomenico SD, Di Gioacchino M, Cuccurullo F, Mezzetti A: Circulating monocyte chemoattractant protein-1 and early development of nephropathy in type 1 diabetes. Diabetes Care 2002;25:1829-1834.

34 Upadhyay A, Larson MG, Guo CY, Vasan RS, Lipinska I, O’Donnell CJ, Kathiresan S, Meigs JB, Keaney JF Jr, Rong J, Benjamin EJ, Fox CS: Inflammation, kidney function and albuminuria in the framingham offspring cohort. Nephrol Dial Transplant 2011;26: 920-926.

35 Tunon J, Blanco-Colio L, Cristobal C, Tarin N, Higueras J, Huelmos A, Alonso J, Egido J,
Asensio D, Lorenzo O, Mahillo-Fernandez I, Rodriguez-Artalejo F, Farre J, Martin-Ventura JL, Lopez-Bescos L: Usefulness of a combination of monocyte chemoattractant protein-1, galectin- 3 , and $\mathrm{n}$-terminal probrain natriuretic peptide to predict cardiovascular events in patients with coronary artery disease. Am J Cardiol 2014;113:434-440.

36 Piemonti L, Calori G, Mercalli A, Lattuada G, Monti P, Garancini MP, Costantino F, Ruotolo G, Luzi L, Perseghin G: Fasting plasma leptin, tumor necrosis factor-alpha receptor 2 , and monocyte chemoattracting protein 1 concentration in a population of glucose-tolerant and glucose-intolerant women: impact on cardiovascular mortality. Diabetes Care 2003;26:2883-2889.

37 Koeda Y, Nakamura M, Tanaka F, Onoda T, Itai K, Tanno K, Ohsawa M, Makita S, Ishibashi Y, Koyama T, Yoshida Y, Omama S, Ogasawara K, Ogawa A, Kuribayashi T, Okayama A: Serum c-reactive protein levels and death and cardiovascular events in mild to moderate chronic kidney disease. Int Heart J 2011;52:180-184.

38 Betriu A, Martinez-Alonso M, Arcidiacono MV, Cannata-Andia J, Pascual J, Valdivielso JM, Fernandez E, Investigators from the NS: Prevalence of subclinical atheromatosis and associated risk factors in chronic kidney disease: the nefrona study. Nephrol Dial Transplant 2014;29:1415-1422.

39 Recio-Mayoral A, Banerjee D, Streather C, Kaski JC: Endothelial dysfunction, inflammation and atherosclerosis in chronic kidney disease - a cross-sectional study of predialysis, dialysis and kidney-transplantation patients. Atherosclerosis 2011;216:446451.

40 Mathew RO, Bangalore S, Lavelle MP, Pellikka PA, Sidhu MS, Boden WE, Asif A: Diagnosis and management of atherosclerotic cardiovascular disease in chronic kidney disease: a review. Kidney Int 2016;91:797807.

41 Levey AS, Stevens LA, Schmid CH, Zhang YL, Castro AF 3rd, Feldman HI, Kusek JW, Eggers P, Van Lente F, Greene T, Coresh J; CKDEPI (Chronic Kidney Disease Epidemiology Collaboration): A new equation to estimate glomerular filtration rate. Ann Intern Med 2009;150:604-612. 